\title{
On the interaction of wind and steep gravity wave groups using Miles' and Jeffreys' mechanisms
}

\author{
J. Touboul ${ }^{1}$, C. Kharif ${ }^{2}$, E. Pelinovsky ${ }^{3}$, and J.-P. Giovanangeli ${ }^{2}$ \\ ${ }^{1}$ Laboratoire de Sondages Electromagnétiques de l'Environnement Terrestre, Toulon, France \\ ${ }^{2}$ Institut de Recherche sur les Phénomènes Hors Equilibre, Marseille, France \\ ${ }^{3}$ Institute of Applied Physics, Nizhny Novgorod, Russia
}

Received: 18 June 2008 - Revised: 14 October 2008 - Accepted: 4 November 2008 - Published: 16 December 2008

\begin{abstract}
The interaction of wind and water wave groups is investigated theoretically and numerically. A steep wave train is generated by means of dispersive focusing, using both the linear theory and fully nonlinear equations. The linear theory is based on the Schrödinger equation while the nonlinear approach is developed numerically within the framework of the potential theory. The interaction between the chirped wave packet and wind is described by the Miles' mechanism. The differences between both approaches are discussed, and the influence of nonlinearity is emphasized. Furthermore, a different mechanism is considered, described by the modified Jeffreys' sheltering theory. From comparison between the two mechanisms, it is found that the persistence of the steep wave group depends on the physical model used, and is significantly increased when we use the latter mechanism.
\end{abstract}

\section{Introduction}

The problem of modelling the interaction of wind and sea waves has been widely studied during the last century. A large number of theories have been proposed to describe the phenomenon. None of them were completely satisfying before the theory derived by Miles (1957), as pointed out by Ursell (1956). The popular Miles' theory is based on the resonant interaction of a sheared air flow with a weakly nonlinear periodic wave field. This theory, complementary to the theory proposed by Phillips (1957), was the first to predict an exponential growth of waves corresponding to the growth observed. By assuming the pressure to vary in phase with the wave slope, Miles correlated the wave growth to

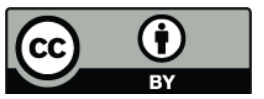

Correspondence to: J. Touboul (julien.touboul@univ-tln.fr) the shear profile of the wind above. Considering the linear stability analysis of this parallel shear flow, he showed that the principal parameter controlling the growth rate was the curvature of the mean wind vertical profile at the critical height. This growth rate was then parameterized by using the wave age $\left(c / u^{*}\right), c$ being the wave phase velocity, and $u^{*}$ the wind friction velocity. Since then, this mechanism as been widely studied and improved, for example through incorporating Reynolds stresses originally ignored in the model (Miles, 1996, 1999). It is now recognized as an excellent description of the wind-wave interaction (Janssen, 2004).

However, the specific case of wind interacting with space limited wave groups was not the central point of the previous investigations. This situation changed recently, with the growing interest of the scientific community in rogue waves. The increasing number of accidents related to those waves lead oceanographers to focus on the study of such events. As suggested by Kharif and Pelinovsky (2003), rogue waves can appear in various places, and are related to numerous physical phenomena. Especially, such waves can appear in storm areas, where they encounter strong winds. In this context, the influence of wind on such unusual waves became a key point.

Recent work by Touboul et al. (2006), Touboul and Kharif (2006), and Kharif et al. (2008) investigated experimentally and numerically the influence of wind on focusing wave groups. Their study emphasized the existence of a strong asymmetry between the focusing and defocusing stages, due to the interaction between wind and very steep waves. In the absence of wind the increase (during the focusing stage) and the decrease (during the defocusing stage) of the maximum magnitude of the wave group envelope present symmetric spatial evolutions. In the presence of wind the symmetry of the spatial evolutions is broken. In relation to the case

Published by Copernicus Publications on behalf of the European Geosciences Union and the American Geophysical Union. 
without wind, the maximum magnitude of the wave group envelope during the defocusing stage is increased under wind action. The previous authors suggested that the process could be described by a modified Jeffreys' sheltering mechanism. This mechanism, first introduced by Jeffreys (1925), is based on the difference of pressure between the leeward and windward faces of the waves induced by air flow separation over high wave crests. With this assumption, they considered that the local air flow separation observed over steep crests was predominant to describe the wind-wave interaction in the presence of steep waves events.

In the framework of wind interaction with steep waves, one should recall that very steep waves are short-lived events in a wave group presenting low steepness' value most of the time. Hence, it is questionable to consider the role of air flow separation as a dominant mechanism. One can wonder if Miles' mechanism could be relevant to describe this specific interaction, since it should act during the whole lifetime of the group. Present work has been motivated by this remark.

In a first step, a linear model based on the Schrödinger equation is derived, describing the evolution of a chirped wave packet under wind forcing. In a second step, full nonlinearity of hydrodynamical equations is introduced to propagate the chirped wave packet under wind action. In both cases, wind is introduced through a linear pressure term, supposed to be in phase with the wave slope. The magnitude of this term depends on a growth parameter. This parameter was chosen accordingly to the theoretical studies of Miles (1957, 1996, 1999), and experimental observations (Komen et al., 1994). Theoretical and numerical results obtained with both models are then compared. The role of nonlinearity is emphasized. The asymmetry induced by this process is then quantified and discussed. Values of asymmetry obtained do not explain experimental observation presented in Kharif et al. (2008). Thus, a comparison with the modified Jeffreys' sheltering mechanism is finally provided and discussed.

\section{Theoretical model}

The spatio-temporal evolution of the envelope of narrowbanded weakly nonlinear water waves may be described by the nonlinear Schrödinger equation (NLS), initially derived in the context of water waves by Benney and Newell (1967). A balance between dispersion and nonlinearity results in this universal equation that reads

$i\left(\frac{\partial A}{\partial t}+c_{g} \frac{\partial A}{\partial x}\right)-\frac{\varepsilon \omega_{0}}{8 k_{0}^{2}} \frac{\partial^{2} A}{\partial x^{2}}-\frac{\varepsilon \omega_{0} k_{0}^{2}}{2}|A|^{2} A=0$,

where $A$ is the complex amplitude. The small parameter of nonlinearity $\varepsilon$ corresponds to the wave steepness, and the surface elevation $\eta(x, t)$ is given by

$\eta(x, t)=\frac{1}{2} A(x, t) \exp \left[i\left(k_{0} x-\omega_{0} t\right)\right]+c . c$.
The wavenumber and frequency of the carrier wave are $k_{0}$ and $\omega_{0}$ respectively, $c_{g}=(d \omega / d k)_{k_{0}}$ is the group velocity and c.c. denotes the complex conjugate. The complex amplitude is assumed to be a slowly varying function of $x$ and $t$. Introducing the transformation $\tau=\omega_{0}\left(t-x / c_{g}\right), y=\varepsilon k_{0} x$, $a=k_{0} A$, as used by Kit and Shemer (2002), and wind forcing in Eq. (1) yields to the forced spatial nonlinear Schrödinger equation

$i \frac{\partial a}{\partial y}-\frac{\partial^{2} a}{\partial \tau^{2}}-|a|^{2} a=i \delta a$.

The RHS term represents the wind action, where $\delta$ is the spatial growth rate. It was initially introduced in an ad hoc manner by Trulsen and Dysthe (1992) to express wind influence. However, this term can also be obtained by expressing the dynamic boundary condition in the presence of wind. Writing the kinematic boundary condition, with the assumption $\delta \simeq \varepsilon^{2}$, provides a link between the pressure term $p$ and the vertical component of the velocity ika. Within the framework of water waves, a proper derivation of the forced NLS equation is developed by Leblanc (2007).

Let us consider the focusing of a linear wave group under wind action. Equation (3) reduces to the following linear parabolic equation

$i \frac{\partial a}{\partial y}=\frac{\partial^{2} a}{\partial \tau^{2}}+i \delta a$,

By introducing $a(\tau, y)=b(\tau, y) \exp (\delta y)$ into equation (4), this equation rewrites

$i \frac{\partial b}{\partial y}=\frac{\partial^{2} b}{\partial \tau^{2}}$.

Equation (5) is generally used to describe the wave focusing of chirped wave trains (see Clauss, 1999; Kharif and Pelinovsky, 2003), since it admits the following solution

$b(\tau, y)=\frac{B_{1}}{\sqrt{1-4 i \Omega_{1}^{2} y}} \exp \left(-\frac{\Omega_{1}^{2} \tau^{2}}{1-4 i \Omega_{1}^{2} y}\right)$.

The corresponding amplitude is given by

$$
|b(\tau, y)|=\frac{B_{1}}{\left(1+16 \Omega_{1}^{4} y^{2}\right)^{\frac{1}{4}}} \exp \left(-\frac{\Omega_{1}^{2} \tau^{2}}{1+16 \Omega_{1}^{4} y^{2}}\right),
$$

and the related phase is

$$
\arg [b(\tau, y)]=-\frac{\operatorname{atan}\left(4 \Omega_{1}^{2} \mathrm{y}\right)}{2}-\frac{4 \Omega_{1}^{4} \tau^{2} y}{1+16 \Omega_{1}^{4} y^{2}} .
$$

The maximum of amplitude, reached for $\tau=0$, decreases as $y^{-1 / 2}$. The frequency modulation, $\Omega(\tau, y)=\partial \arg (b) / \partial \tau$, varies linearly with time in the wave train. Hence, the low frequency oscillations are located ahead of the wave group as it is expected for a dispersive system. Under the transformation $y \rightarrow-y$ the high frequency oscillations are now located 
in front of the wave train (see Fig. 1). The dispersive behavior of water waves leads this modulated wave group to focus energy in time and space. A caustic is formed, corresponding to a large amplitude wave. It describes the dispersive focusing of a chirped wave packet, generating an extreme wave event of maximal amplitude $B_{1}$ and characteristic spectral width $\Omega_{1}$, at point $y=0$.

Let us assume the wave maker located at $y=-X_{f}$, and introduce the new spatial coordinate $z=y / L+1$. Here, $L=k_{0} X_{f}$ is a dimensionless focusing length with $X_{f}$ the dimensional coordinate of the focus point. Let us also introduce $q=4 \Omega_{1}^{2} L$, the phase index. The amplitude Eq. (7) of solution Eq. (6) reads now

$$
\begin{aligned}
|b(\tau, z)|=B_{0} & \left(\frac{1+q^{2}}{1+q^{2}(z-1)^{2}}\right)^{1 / 4} \times \\
& \exp \left(-\Omega_{0}^{2} \tau^{2} \frac{1+q^{2}}{1+q^{2}(z-1)^{2}}\right),
\end{aligned}
$$

while the argument Eq. (8) becomes

$$
\begin{aligned}
\arg [b(\tau, z)]= & \frac{\operatorname{atan}(\mathrm{q}(\mathrm{z}-1))}{2}+ \\
& \left(\frac{1+q^{2}}{1+q^{2}(z-1)^{2}}\right) q(z-1) \Omega_{0}^{2} \tau^{2} .
\end{aligned}
$$

In Eqs. (9) and (10), $B_{0}$ and $\Omega_{0}$ refer to initial amplitude and characteristic spectral width respectively. They are related to $B_{1}$ and $\Omega_{1}$ through

$$
B_{0}=\frac{B_{1}}{\left(1+q^{2}\right)^{1 / 4}} \quad \text { and } \quad \Omega_{0}=\frac{\Omega_{1}}{\left(1+q^{2}\right)^{1 / 2}} .
$$

The linear evolution of a transient wave packet yielding a steep wave event is completely described by Eqs. (9) and (10), which only depend on three independent parameters: the initial maximum amplitude $B_{0}$, the initial characteristic spectral width $\Omega_{0}$, and the phase index $q$.

As mentioned earlier, wind effect is introduced by multiplying solution Eq. (9) by exp( $\delta y)$. Maximum amplitude of the envelope of the chirped wave packet propagated under the action of wind is given by

$A(z)=A_{0}\left(\frac{1+q^{2}}{1+q^{2}(z-1)^{2}}\right)^{1 / 4} \exp (\gamma z)$,

where $A_{0}=B_{0}$ is the maximum amplitude of the initial condition's envelope, and $\gamma=\delta L$ is the dimensionless spatial growth rate of wave energy. It is clear that the wave amplitude $A(z)$ is now non-symmetric around focusing point $z=1$, while it was in the absence of wind.
The spatial growth rate of energy is computed to obtain an exponential growth of the waves corresponding to Miles' theory. Hence, the spatial growth rate of wave amplitude due to a wind of velocity $U$ is given by

$\delta=\frac{\beta}{\kappa^{2}} \frac{\rho_{a}}{\rho_{w}}\left(\frac{u^{*}}{c}\right)^{2}$.

In Eq. (13), $\beta$ is the energy-transfer parameter of Miles, $\kappa=0.4$ is the Von Karman constant, $\rho_{a}$ and $\rho_{w}$ are the densities of air and water respectively, $u^{*}=\sqrt{C_{d}} U$ is the friction velocity, $c$ is the wave phase velocity and $k_{0}$ is the corresponding wave number. The drag coefficient $C_{d}=0.004$ is known experimentally for such wave groups, and for inverse wave age $u^{*} / c=0.2$. These experiments are described in Kharif et al. (2008), and correspond to an averaged frequency of $1 \mathrm{~Hz}$ for the chirped wave packet propagated under a wind velocity of $5 \mathrm{~m} / \mathrm{s}$. The value of $\beta$ is considered as a parameter, as it will be discussed later. Phase velocity and wave number are obtained using the linear dispersion relation in infinite depth: $k_{0}=\omega_{0}^{2} / g$, and $c=g / \omega_{0}$.

\section{Numerical model}

The focusing wave group has low steepness during the major part of its evolution. This feature can justify the use of the linear approach. Nevertheless, the steepness reached in the vicinity of the focusing point becomes important, and one should consider nonlinear effects due to large steepness. Hence, the fully nonlinear potential equations governing the free surface motion are considered here to describe the evolution of the transient wave group. These equations are the Laplace equation and nonlinear boundary conditions

$$
\begin{aligned}
& \Delta \phi=0, \quad \text { for } \quad-h<z<\eta(x, t), \\
& \frac{\partial \eta}{\partial t}+\frac{\partial \eta}{\partial x} \frac{\partial \phi}{\partial x}-\frac{\partial \phi}{\partial z}=0, \quad \text { on } \quad z=\eta(x, t), \\
& \frac{\partial \phi}{\partial t}+\frac{1}{2} \nabla \phi \cdot \nabla \phi+g \eta=-\frac{p}{\rho_{w}}, \quad \text { on } \quad z=\eta(x, t), \\
& \frac{\partial \phi}{\partial n}=0 \quad \text { on } \quad z=-h,
\end{aligned}
$$

where $\phi(x, z, t)$ is the velocity potential, $z=\eta(x, t)$ is the equation of the surface, $g$ is the acceleration due to gravity, $p$ is the atmospheric pressure at the surface, $x$ and $z$ are the horizontal and vertical coordinates respectively and $t$ is the time. The numerical wave tank is closed using wall conditions on its ends. One of these ends is mobile and used as a wavemaker. The equations are solved with a boundary integral element method (BIEM), using a mixed Euler Lagrange description of the above equations. Time stepping is performed using a fourth order Runge and Kutta scheme, with a constant time step. More details can be found in Touboul et al. (2006). 


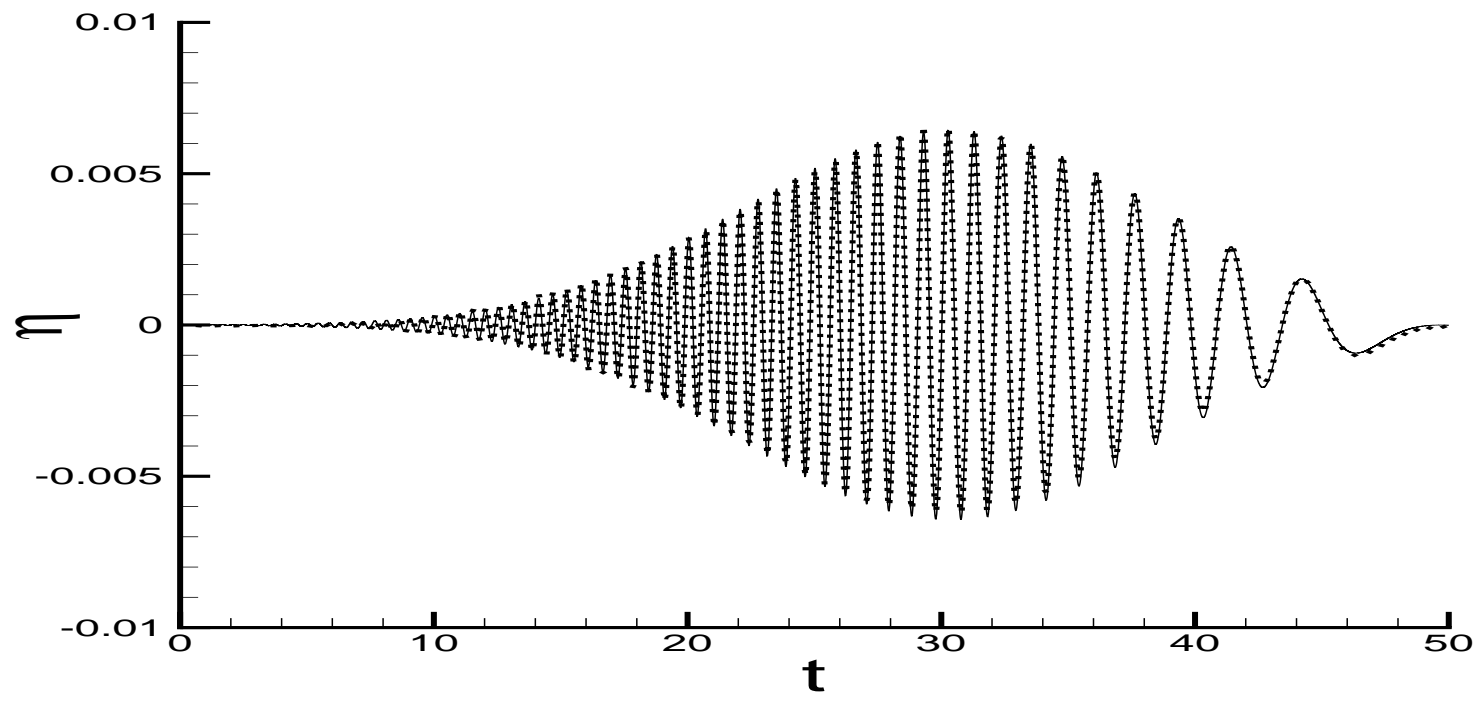

Fig. 1. Surface elevation $\eta(m)$ as a function of time $t(s)$ corresponding to initial condition 4 of Table 1: theoretical solution (-) and numerical simulation $(\cdots)$.

Table 1. Initial conditions used for the simulations and corresponding maximal steepness.

\begin{tabular}{ccccc}
\hline & $B_{1}$ & $\varepsilon_{t h}$ & $|\partial \eta / \partial x|$ & $\varepsilon_{s}$ \\
\hline 1 & 0.075 & 0.024 & 0.030 & 0.030 \\
2 & 0.150 & 0.048 & 0.061 & 0.061 \\
3 & 0.225 & 0.072 & 0.098 & 0.098 \\
4 & 0.300 & 0.095 & 0.131 & 0.130 \\
5 & 0.375 & 0.119 & 0.181 & 0.178 \\
6 & 0.450 & 0.144 & 0.227 & 0.220 \\
7 & 0.525 & 0.168 & 0.305 & 0.289 \\
\hline
\end{tabular}

The wind effect is described by the pressure term $p(x, t)$ applied at the interface, in Eq. (16). Following Banner and Song (2002), this term is assumed to be in phase with the wave slope. The surface pressure is assumed to have a distribution of the form

$p(x, t)=\alpha \rho_{a} u^{*^{2}} \frac{\partial \eta}{\partial x}$,

where $\rho_{a}$ is the air density, and $\alpha$ an unknown parameter. The total energy input from this pressure term is $\int_{\lambda}(p \partial \eta / \partial t) d x$, where $\lambda$ is a wavelength. Thus, the relation between $\alpha$ and the spatial growth rate is easily established by using a linear description of $\eta(x, t)$, and calculating this integral. It comes

$\delta=\frac{\beta}{\kappa^{2}} \frac{\rho_{a}}{\rho_{w}}\left(\frac{u^{*}}{c}\right)^{2}=\frac{\alpha}{2} \frac{\rho_{a}}{\rho_{w}}\left(\frac{u^{*}}{c}\right)^{2}$.

The simulations are conducted using the pressure term of Eq. (18), with the value $\alpha=2 \beta / \kappa^{2}$. The growth rate due to this pressure term is equal to the theoretical growth rate. However, it is important to notice that full nonlinearity is only introduced in water. This description of air flow, based on Miles' theory, neglects nonlinearity. The friction velocity $u^{*}$ is assumed constant along the group, which is a basic assumption. However, the recent work by Makin et al. (2007) emphasizes that the variation of $u^{*}$ in the range of steepness considered in our groups is of order $10 \%$.

\section{Results and discussion}

Effect of nonlinearity on transient wave packets has already been investigated by several authors (Brown and Jensen, 2001; Shemer et al., 2007). These authors observed a front - tail asymmetry appearing on the wave group envelope. In these studies, the authors emphasized that this asymmetry was correlated to low order nonlinearity. It was explained as soon as bound waves were taken into account. In both cases, they found that the maximum amplitude of the wave group envelope, or amplification factor, had a nonlinear behavior around the focusing point. However, results fitted with linear theory far from the focusing point, this maximum presenting a symmetrical behavior around the focusing point. In the following we focus on the asymmetry induced in this amplification factor for wave trains propagating under wind action.

To investigate the effect of nonlinearity, several initial conditions are used. Table 1 presents these conditions, with the corresponding nonlinearity reached during the simulations. $\varepsilon_{t h}=B_{1} \times k_{0}$ is the maximum steepness at the focusing point, given by the linear theory. $|\partial \eta / \partial x|$ is the maximum local slope obtained from nonlinear simulations without wind, while $\varepsilon_{s}$ is the steepness of a high order nonlinear Stokes wave, computed with the method developed by LonguetHiggins (1987), presenting the same maximal slope. One 
should note that the linear theory underestimates steepness up to $70 \%$.

Initial conditions correspond to initial parameters $X_{f}=15 \mathrm{~m}$ and $\Omega_{1}=0.3$. The frequency of the carrier wave is chosen such as $\omega_{0}=2 \pi \mathrm{rad} / \mathrm{s}$, which is similar to the value used in Kharif et al. (2008). Figure 1 shows the theoretical initial elevation (solid line) versus time, obtained from Eqs. (2) and (9), at $z=0$. It corresponds to the initial focusing wave train, with initial parameter $B_{1}=0.3$. Figure 1 displays the nonlinear free surface elevation (dotted line) too, recorded one carrier wavelength away from the paddle. It has been obtained iteratively, to reproduce theoretical initial condition. This probe location is considered as being the origin of the $x$ axis in the following simulations. The comparison between both theoretical and numerical probes shows an excellent agreement.

These initial conditions are used to propagate numerically wave groups without wind, and under wind, for $u^{*} / c=0.2$. For this value of inverse wave age, several values of $\alpha$ extending from 27 and 38 are available in the literature (see Fig. 1 of Banner and Song, 2002, cited from Komen et al., 1994). Banner and Song (2002) used $\alpha=32.5$. According to Eq. (19), this value corresponds to $\beta=2.6$. Simulations are then performed with $\beta=2.2, \beta=2.6$ and $\beta=3$, the latter value corresponding to the value obtained by Miles (1996). The results are then compared.

Figure 2 shows the amplification factor $A / A_{0}$ versus dimensionless fetch $z$. Amplification parameter is defined numerically by

$\frac{A}{A_{0}}(z)=\frac{\max \{\eta(z, t)\}}{\max \{\eta(0, t)\}}$.

Figure 2 a corresponds to the theoretical solution and numerical solution corresponding to the initial condition 4 of Table 1, without wind. Nonlinear effects results in a widening of the curve around the peak. The nonlinear interactions among the different components of the group produce a detuning effect that diminishes the peak height. The maximum amplitude is weaker in the nonlinear simulation than predicted by the linear theory, confirming results obtained by Shemer et al. (2007).

Figure $2 b$ shows the spatial evolution of the numerical solution corresponding to initial condition 4 of Table 1 and theoretical solutions with and without wind. The growth rate used herein is $\beta=2.6$. A comparison between theoretical linear solutions emphasizes the effect of the wind. An increase of the amplification factor and a weak asymmetry between focusing and defocusing stages are observed. These features are more important when the nonlinearity is introduced.

In both Fig. $2 \mathrm{a}$ and $\mathrm{b}$, oscillations of the amplification factor appear around the peak. Figure 3 presents an enlargement of these figures, where these oscillations can be seen. One can notice that the oscillations present a wave number equal to $k_{0}$. They are amplified in the presence of wind. Similar
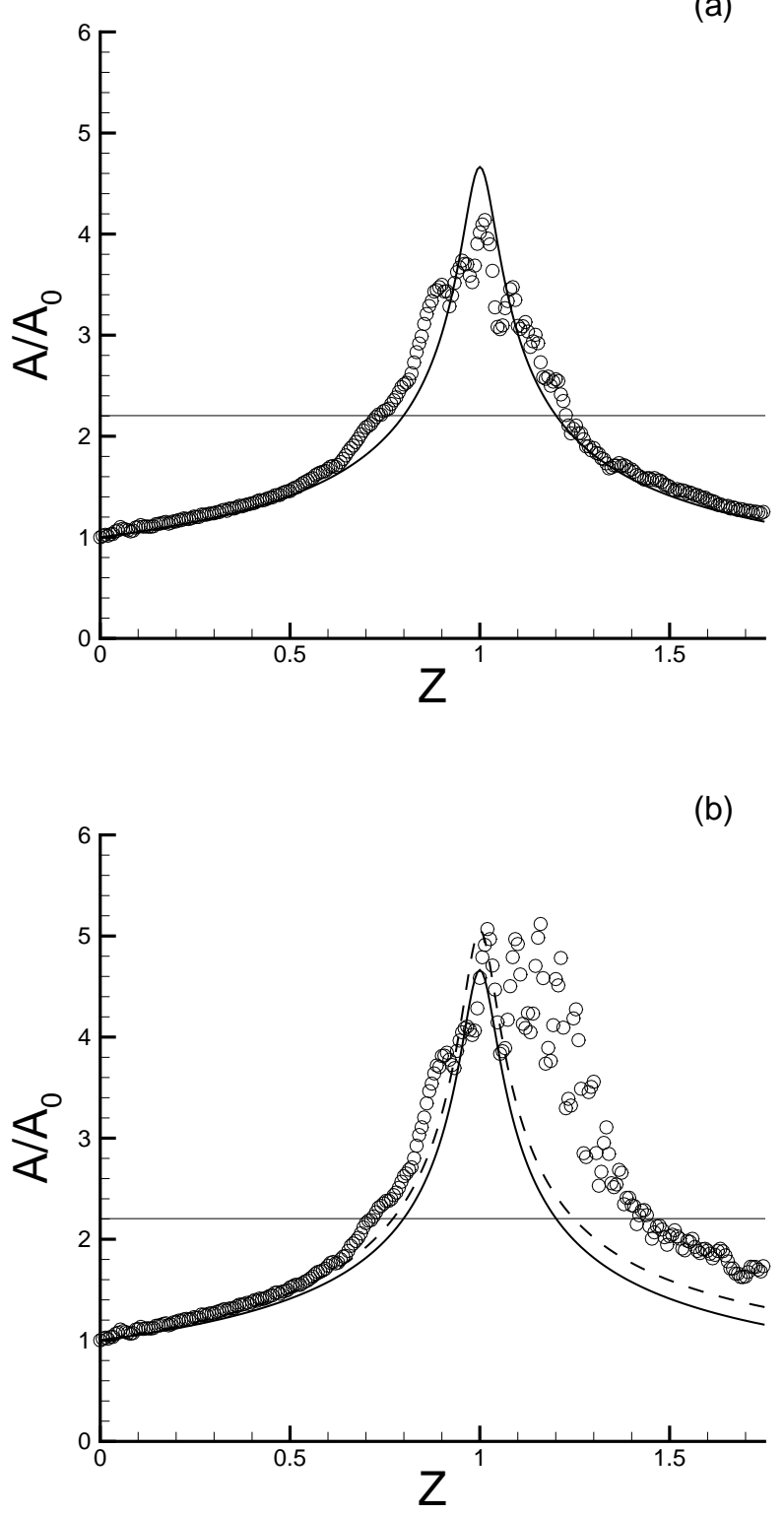

Fig. 2. (a) Amplification factor $A / A_{0}(z)$ for a transient wave group propagated without wind. (-): Theoretical linear solution; (o) Numerical solution. (b) Amplification factor for a wave group propagated under wind action, with growth rate $\beta=2.6$. (-): Theoretical linear solution without wind; $(--)$ : Theoretical linear solution with wind; (o): Numerical solution. Both simulations are conducted with initial condition 4 of Table 1 .

oscillations have already been observed by Song and Banner (2002) around the maximum of modulation for a wave group submitted to Benjamin-Feir instability, without wind. They noticed that these oscillations were the consequence of the asymmetry between wave crests and troughs. When a crest or a trough are located at the maximum envelope amplitude, the densities of energy are not the same. It results in 

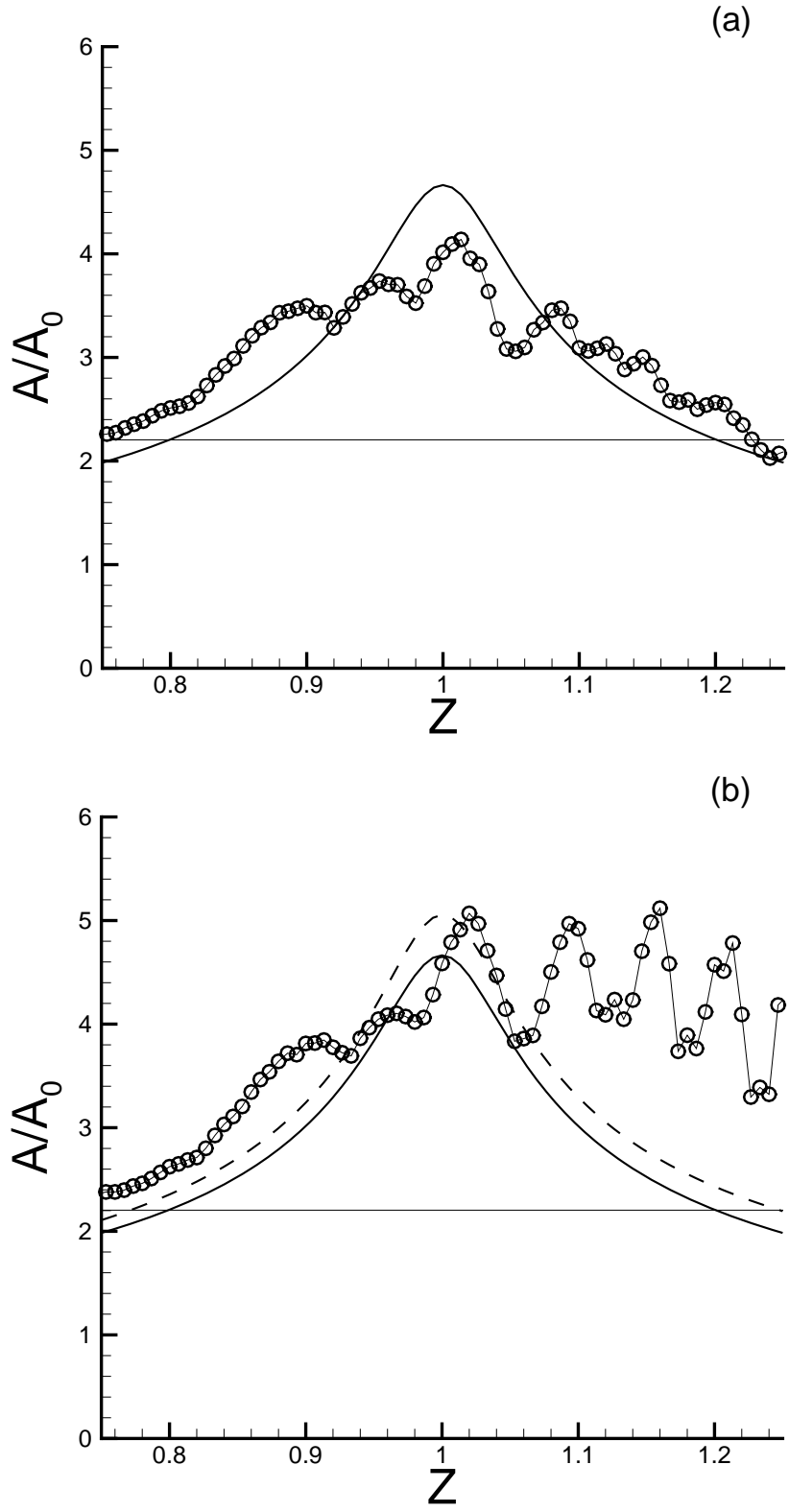

Fig. 3. Enlargement of Fig. 2 around focusing point.

an oscillation of frequency $2 \omega_{0}$, and wave number $k_{0}$, of the maximum amplitude of the envelope of the group. This result was also observed experimentally by Shemer et al. (2007) in the framework of linear focusing. By comparing their results to computations based on Zakharov equation, they emphasized that the asymmetry between wave crests and troughs was correlated to the dominant role played by bound waves associated to the leading wave. Thus, this phenomenon is correlated to nonlinearity, rather than wind action. However, it is reasonable to consider that wind increases nonlinearity. The phenomenon observed is similar to the one described by Song and Banner (2002) and Shemer et al. (2007).
Figures $2 \mathrm{a}, \mathrm{b}, 3 \mathrm{a}$ and $\mathrm{b}$ also display an horizontal line, which corresponds to $A / A_{0}=2.2$. A wave is considered to be a rogue wave as soon as $A / A_{0} \geq 2.2$, as mentioned by Kharif and Pelinovsky (2003). This criterion is used to define a significant length of existence of steep waves in the group. This length $L_{f}$ during which this criterion is satisfied, depends on the asymmetry of the focusing-defocusing curve.

Figure $4 \mathrm{a}$ and $\mathrm{b}$ show respectively the length $L_{f}$, normalized by its value without wind $L_{f 0}$, and the maximum amplitude $A_{f}$ reached in the transient wave packet during the focusing-defocusing process, normalized by the corresponding value without wind $A_{f 0}$, for several values of the growth rate $\beta$. Both quantities are presented as a function of the steepness parameter $\varepsilon_{s}$. This nonlinear parameter used as abscissa is the steepness presented in Table 1. It corresponds to an estimate of the steepness reached in the simulations without wind. The value $\varepsilon_{s}=0$ corresponds to the theoretical solution, and the corresponding points on Fig. $4 \mathrm{a}$ and $\mathrm{b}$ show respectively the normalized length $L_{f}$ and the normalized amplitude $A_{f}$ calculated from the theoretical approach. Simulations have been performed with growth rates $\beta=2.2$, $\beta=2.4$, and $\beta=3$ respectively. Points corresponding to larger steepness are not presented, because wave breaking occurred during these simulations. It is important to emphasize that the value of the local slope $|\partial \eta / \partial x|$ in the numerical simulations in the presence of wind can be larger than 0.5 .

One can notice from Fig. 4a that nonlinearity plays a significant role in sustaining steep wave groups. For small value of the growth rate $\beta=2.2$, the deviation from the linear theory is not very important (about 10\%). For larger values of $\beta$, 2.6 and 3 , the deviation from the linear theory is quite more significant (up to $50 \%$ ). For the latter cases, wind input is more important, and nonlinearity is increased. The transient wave packet which is affected by nonlinearity, presents steep waves over significant distances.

From Fig. $4 \mathrm{~b}$, it is observed that the normalized amplification $A / A_{0}$ is not significantly affected by the nonlinear parameter $\varepsilon_{s}$. In every simulations, the deviation from the linear theory has never been larger than $13 \%$. This confirms the fact that nonlinear interactions between waves lead to the detuning process mentioned above.

However, as mentioned in Sect. 3, nonlinearity in the air flow is not taken into account using this mechanism. Its relevance to describe the interaction of wind and steep waves might be questionable. The Jeffreys' sheltering mechanism describes air flow separation over waves. This mechanism is not relevant for low steepness waves as shown by Stanton et al. (1932). However, for larger steepness, it is well known that air flow separation occurs, resulting in a significant increase of wind to wave energy flux. Belcher and Hunt (1998) suggested that the Jeffreys sheltering mechanism would be appropriate to describe wind forcing over the steepest waves. This behavior can be described by introducing a threshold in 
slope, and expressing the pressure term of Eq. (18) by

$$
\begin{cases}p(x)=0 & \text { if }|\partial \eta / \partial x|_{\max }<|\partial \eta / \partial x|_{c} \\ p(x)=\rho_{a} s & (U-c)^{2} \frac{\partial \eta}{\partial x}(x) \\ & \text { if }|\partial \eta / \partial x|_{\max } \geq|\partial \eta / \partial x|_{c}\end{cases}
$$

where $s$ is a sheltering coefficient, introduced by Jeffreys (1925). By introducing the rates of growth of wave energy due to wind action, $\gamma_{\text {Jeffreys }}$ and $\gamma_{\text {Miles }}$, corresponding to the Jeffreys' sheltering and Miles' mechanisms respectively, one can express

$$
\frac{\gamma_{\text {Jeffreys }}}{\gamma_{\text {Miles }}}=\frac{s \kappa^{2}}{\beta C_{d}}\left(1-\frac{c}{U}\right)^{2} \text {, }
$$

Within the framework of our simulations, it is found that this ratio always exceeds three, meaning that the characteristic time scale of the Miles' mechanism is more than three times larger than the characteristic time scale of the Jeffreys' sheltering mechanism. More details about the modified Jeffreys' sheltering mechanism can be found in Touboul et al. (2006).

In order to compare Miles' theory with the modified Jeffreys' sheltering mechanism, simulations have also been performed using this latter phenomenon. Each initial condition has been propagated under the Jeffreys' sheltering mechanism. The parameter $|\partial \eta / \partial x|_{c}$ was chosen to be $60 \%$ of the maximum value presented in Table 1 , while the sheltering coefficient was chosen to be $s=0.5$, as suggested by Jeffreys (1925), and confirmed experimentally. The numerical and theoretical spatial evolutions of the amplification factor $A / A_{0}(z)$ are plotted in Fig. 5. The solution computed numerically from the fully nonlinear equations corresponds to the initial condition 7 of Table 1 under wind action when the modified Jeffreys' sheltering mechanism is used. The theoretical solution given by the linear theory without wind effect and the horizontal straight line corresponding to the rogue wave criterion are also plotted, for the sake of reference.

Jeffreys pressure term is applied on the surface of each wave of the group overcoming this threshold. It is the critical parameter $|\partial \eta / \partial x|_{c}$ mentioned above. Thus, during the focusing-defocusing process, the modified Jeffreys' sheltering mechanism is only active near the focusing point. This is very different from Miles' mechanism, which is effective during the whole process. The total amount of energy transferred from wind to waves is larger through Jeffreys' mechanism during extreme wave event, but the energy distribution in time and space is different from a mechanism to another. This changes considerably the dynamics of the chirped wave packets under wind action. In the absence of wind, wave groups of large steepness are near breaking in the vicinity of the focusing point. In the presence of wind, some energy is added. Using Miles' mechanism, a large amount of energy have already been transferred before occurrence of the extreme wave event. With Jeffreys' mechanism, the
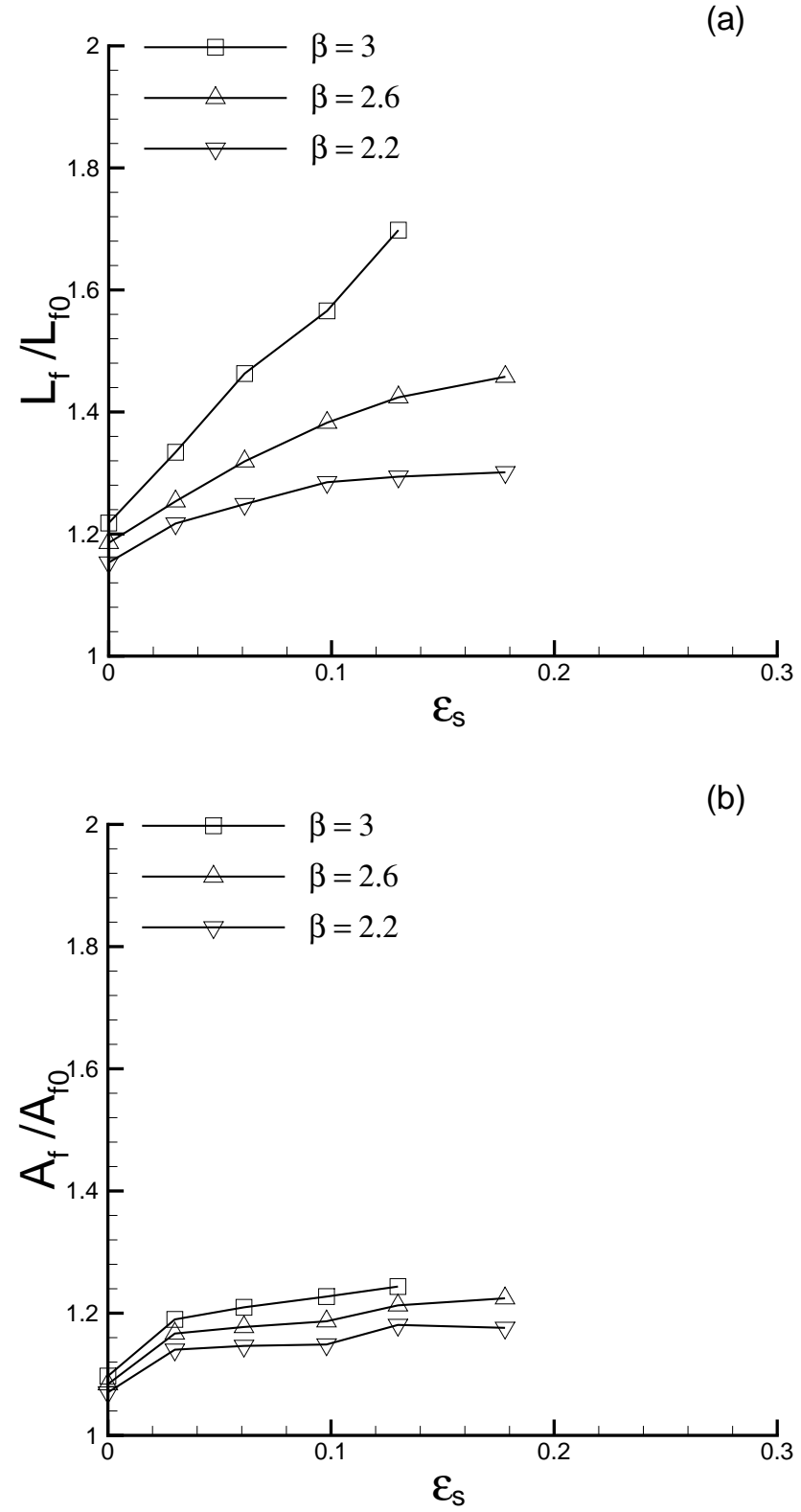

Fig. 4. (a): Length of existence of the steep wave under wind action $L_{f} / L_{f 0}$ as a function of the nonlinear parameter $\varepsilon_{s}$. (b): Maximum amplitude reached by the transient wave group under wind action versus nonlinear parameter $\varepsilon_{s}$. The value $\varepsilon_{s}=0$ corresponds to theoretical model in both cases.

transfer starts when the chirped wave packet reaches the focusing point. If wind is introduced using the Miles' mechanism, this would result in the disappearance of the group close to that point because breaking will occur. It is not the case for wave groups propagated using Jeffreys' sheltering mechanism. Results can be seen in Fig. 5. In that case, the length of existence of the rogue wave event is significantly increased (at least 200\%). This result is sensitive to the set of parameters used to model air flow separation. However, 


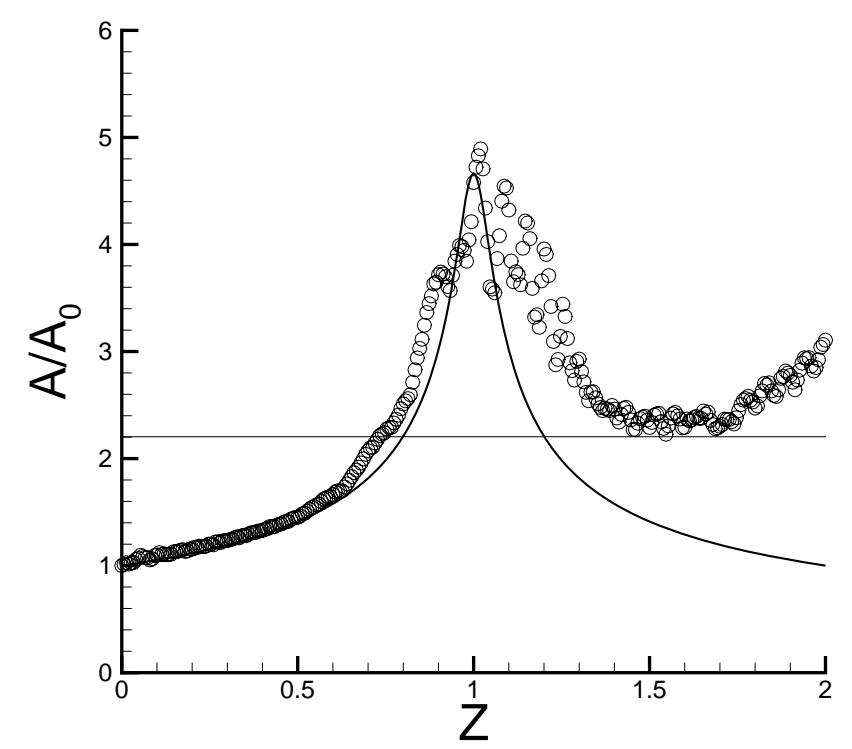

Fig. 5. Amplification factor $A / A_{0}(z)$ for a transient wave group. (-): Theoretical linear solution without wind; (o): Numerical solution corresponding to a wave group of steepness $\varepsilon_{\text {Stokes }}=0.28$ propagated under wind modelled through the modified Jeffreys' sheltering mechanism.

this model produces a persistence of rogue waves which is in good agreement with experimental behavior observed by Kharif et al. (2008).

\section{Conclusions}

The influence of wind on the dynamics of extremely steep waves produced from chirped wave packets has been studied theoretically and numerically. Wind has been introduced through a pressure term acting on the free surface. The growth parameter of this term was chosen accordingly to the quasi-linear Miles' theory, assuming a weak variation of wind stress along the group. The role of nonlinearity in the process has been investigated by comparing the linear Schrödinger equation to numerical simulations, based on the fully nonlinear equations of water waves within the framework of the potential theory.

Results derived from the linear Schrödinger equation point out that the wind is responsible for an increase of the maximum wave amplitude. A weak asymmetry in wave group amplitude during the focusing-defocusing process is also observed.

The nonlinear simulations have partially confirmed these results. Several initial conditions have been used in the numerical wave tank. These initial conditions, corresponding to different values of the steepness $\varepsilon_{s}$, lead to several behaviors. Results are analyzed as a function of the nonlinear parameter $\varepsilon_{s}$. In every simulations, a weak deviation from linear theory for the maximum of amplitude is observed (less than
$13 \%$ ) while it is not the case for the length $L_{f}$ which is proportional to wind input. Major differences are found when considering the asymmetry of wave group amplitude during the focusing-defocusing process. The asymmetry observed in the focusing-defocusing process is significantly larger than expected, resulting in the persistence over larger distances of the extreme wave event. The relative deviation between nonlinear and linear models with wind action presents values up to $50 \%$. However the relative deviation between the nonlinear models with and without wind never exceeds $70 \%$.

Experimentally, Touboul et al. (2006) and Kharif et al. (2008) found an increase of duration length larger than $200 \%$. We can conclude that Miles' mechanism cannot explain correctly experimental observations. Hence, simulations have also been performed using the modified Jeffreys' sheltering theory. In this case, a better agreement between numerical and experimental results is found. The relative deviation between the nonlinear models with and without wind exceeds $200 \%$, for large values of $\varepsilon_{s}$. In spite of its strong hypothesis, this simple wind model is capable of reproducing qualitatively the behavior of the chirped wave packet under wind action.

Acknowledgements. This work was supported by the INTAS Grant No 06-1000013-9236.

Edited by: R. Grimshaw

Reviewed by: two anonymous referees

\section{References}

Banner, M. I. and Song, J.-B.: On determining the onset and strength of breaking for deep water waves. part ii : Influence of wind forcing and surface shear, J. Phys. Oceanogr., 32(9), 25592570, 2002.

Belcher, S. E. and Hunt, J. C. R.: Turbulent flows over hill and waves, Annu. Rev. Fluid. Mech., 30, 507-538, 1998.

Benney, D. J. and Newell, A. C.: The propagation of nonlinear wave envelop, J. Math. Phys., 46, 133-139, 1967.

Brown, M. G. and Jensen, A.: Experiments on focusing unidirectional water waves, J. Geophys. Res., 106(C8), 16917-16928, 2001.

Clauss, G.: Task-related wave groups for seakeeping tests or simulation of design storm waves, Appl. Ocean Res., 21(5), 219-234, 1999.

Janssen, P.: The interaction of ocean waves and wind, Cambridge University Press 2004.

Jeffreys, H.: On the formation of wave by wind, Proc. Roy. Soc. A., 107, 189-206, 1925.

Kharif, C., Giovanangeli, J.-P., Touboul, J., Grare, L., and Pelinovsky, E.: Influence of wind on extreme wave events: Experimental and numerical approaches, J. Fluid Mech., 594, 209-247, 2008.

Kharif, C. and Pelinovsky, E.: Physical mechanisms of the rogue wave phenomenon, Eur. J. Mech. B./Fluids, 22, 603-634, 2003. 
Kit, E. and Shemer, L.: Spatial versions of the zhakharov and dysthe evolution equations for deep water gravity waves, J. Fluid Mech., 450, 201-205, 2002.

Komen, G. J., Cavaleri, L., Donelan, M., Hasselmann, S., Janssen, P. A. E. M.: Dynamics and Modelling of Ocean Waves, Cambridge University Press, 1994.

Leblanc, S.: Amplification of nonlinear surface waves by wind, Phys. Fluids, 19, 101705, doi:10.1063/1.2786002, 2007.

Longuet Higgins, M.: Lagrangian moments and mass transport in Stokes waves, J. Fluid Mech., 179, 547-555, 1987.

Makin, V. K., Branger, H., Peirson, W. L., and Giovanangeli, J.-P.: Stress above wind-plus-paddle waves: modelling of a laboratory experiment, J. Phys. Oceanogr., 37(12), 2824-2837, 2007.

Miles, J. W.: On the generation of surface waves by shear flow, J. Fluid Mech., 3, 185-204, 1957.

Miles, J. W.: Surface-wave generation: a viscoelastic model, J. Fluid Mech., 322, 131-145, 1996.

Miles, J. W.: The quasi-laminar model for wind-to-wave energy transfer, in: Wind-over-wave couplings, edited by: Sajjadi, S. G., Thomas, N. H., and Hunt J. C. R., The institute of mathematics and its applications conference series, 69, 1-7, Clarendon Press, Oxford, 1999.

Phillips, O. M.: On the interaction of waves by turbulent wind, J. Fluid Mech., 2, 417-455, 1957.
Shemer, L., Goulitski, K., and Kit, E.: Evolution of wide spectrum unidirectional wave groups in a tank: an experimental and numerical study, Eur. Journ. Mech. B./Fluids, 26, 193-219, 2007.

Song, J.-B. and Banner, M. I.: On determining the onset and strength of breaking for deep water waves, part i : Unforced irrotational wave groups, J. Phys. Oceanogr., 32(9), 2541-2558, 2002.

Stanton, T., Marshall, D., and Houghton, R.: The growth of waves on water due to the action of the wind, Proc. Roy. Soc. A., 137, 283-293, 1932.

Touboul, J., Giovanangeli, J.-P., Kharif, C., and Pelinovsky, E.: Freak waves under the action of wind: Experiments and simulations, Eur. J. Mech. B./Fluids, 25(5), 662-676, 2006.

Touboul, J. and Kharif, C.: On the interaction of wind and extreme gravity waves due to modulational instability, Phys. Fluids, 18, 108103, doi:10.1063/1.2374845, 2006.

Trulsen, K. and Dysthe, K. B.: Action of windstress and breaking on the evolution of a wavetrain, in: Breaking Waves, IUTAM Symposium Sydney (Australia), edited by: Banner, M. I. and Grimshaw, R. H. J., Springer Verlag Berlin Heidelberg, 1992.

Ursell, F.: Wave generation by wind, in: Surveys in Mechanics, edited by: Batchelor, G. K., Cambridge University Press, 1956. 